\title{
X-ray diffraction line broadening under elastic deformation of a polycrystalline sample: An elastic-anisotropy effect
}

\author{
Anil K. Singh ${ }^{\text {a) }}$ and Chellayyan Balasingh \\ Materials Science Division, National Aerospace Laboratories, Bangalore 560 017, India
}

(Received 16 January 2001; accepted for publication 6 June 2001)

\begin{abstract}
A homogeneous stress field imposed on a polycrystalline sample containing elastically anisotropic crystallites produces an inhomogeneous strain field. The average strain causes a shift of the diffraction-line position, and the variance of the strain is a measure of line broadening. Though the shift of the line is commonly observed, earlier attempts to measure the broadening caused by elastic anisotropy were not conclusive. In the article, expressions have been derived for the average strain and variance of strain for a polycrystalline sample (cubic system) subjected to uniaxial elastic stress. The (310) and (222) lines of beta brass under an uniaxial load have been recorded using Co $K \alpha$ and $\mathrm{Cu} K \alpha$ radiations, respectively. The average strain and variance of strain derived from the measured diffraction line profiles are in good agreement with those predicted by the theory. The present measurements provide conclusive evidence for the diffraction line broadening caused purely by elastic anisotropy. (C) 2001 American Institute of Physics. [DOI: 10.1063/1.1388571]
\end{abstract}

\section{INTRODUCTION}

A homogeneous stress field applied to a polycrystalline sample introduces an inhomogeneous strain field if the crystallites constituting the sample exhibit elastic anisotropy. The $\mathrm{X}$-ray diffraction studies on stressed polycrystalline samples can yield interesting information on the strains in the crystallites. For example, the position of a diffraction line contains the information about average strain along the reciprocal lattice vector, the average being for the group of crystallites that contribute to the diffracted intensity at the point of observation. This aspect has been exploited in the measurement of stresses that arise during the fabrication of components and processing of materials. ${ }^{1-4}$ The diffraction line broadening gives information on the variance of strain. ${ }^{5,6}$ Early investigators ${ }^{7,8}$ had realized that elastic deformation of a polycrystalline sample containing elastically anisotropic crystallites could lead to a broadening of the diffraction line profile. However, these investigators ${ }^{7,8}$ were not successful in detecting such a broadening in their experiments. These observations led to a general conclusion ${ }^{9}$ that the broadening caused by the elastic deformation of a polycrystalline sample was too small to be detected. In subsequent $\mathrm{x}$-ray diffraction studies on polycrystalline samples, this source of broadening did not receive attention.

The theoretical aspects of the line broadening arising from elastic anisotropy, however, continued to attract the attention of theorists. Sayers ${ }^{10}$ carried out a detailed theoretical analysis of the strain field under the elastic deformation of a polycrystalline sample using the methods of Voigt, ${ }^{11}$ Reuss, ${ }^{12}$ and Kröner ${ }^{13}$ and presented detailed calculations on iron. Recent attempts ${ }^{14-24}$ to model the stress state of the sample compressed in a diamond anvil cell (DAC) and to calculate the resulting strains have led to the revival of interest in this field. Funamori et al. ${ }^{25}$ investigated theoretically the line

${ }^{a)}$ Electronic mail: aksingh@css.cmmacs.ernet.in broadening arising from the elastic anisotropy in the case of a triaxial stress state which exists in the sample compressed in a DAC. An analysis of the line broadening arising from the elastic anisotropy of the crystallites is expected to give additional information on the lattice strains. Though the theoretical studies have led to a better understanding of the nature of the broadening, its measurement and a comparison with the theoretical predictions have not yielded conclusive results so far. In this study we have measured the diffraction line profiles of (310) and (222) reflections of beta brass elastically deformed under uniaxial load. For a comparison of the experimental results with the theory, we have derived expressions for the average strain and variance of strain under a uniaxial load. The average strain and variance of strain derived from the line-profile measurements are then compared with the theoretical predictions.

\section{THEORETICAL CONSIDERATIONS}

\section{A Expression for the variance of strain (cubic system)}

In this section we derive the expression for the average and variance of elastic strain in the crystallites of a polycrystalline sample under a uniaxial load. A random distribution of the crystallites in the sample is assumed. The intensity of the diffracted beam (Fig. 1) arises from a suitably oriented group of crystallites ( $\varphi$ group). The reflecting plane normals (reciprocal lattice vectors) of these crystallites lie in the plane defined by the incident and diffracted beams and bisect the angle between the two. The average strain (along the plane normal) of only the $\varphi$ group of crystallites determines the shift of the diffraction line, while the variance of the strain is related to the broadening. ${ }^{5,6}$ The strains calculated under the assumptions of strain continuity ${ }^{11}$ (Voigt assumption) and stress continuity ${ }^{12}$ (Reuss assumption) across the boundaries separating the crystallites represent, respectively, the lower and upper bounds. The strain in an actual case can 


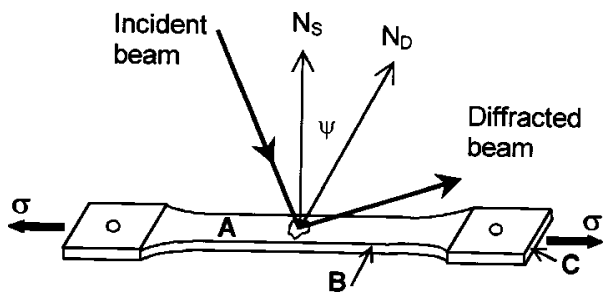

FIG. 1. The schematic view of the test specimen $\left(145 \times 24 \times 0.95 \mathrm{~mm}^{3}\right) . N_{D}$ and $N_{S}$ are the reflecting plane normal and surface normal, respectively.

be represented by a weighted average of these two bounds. We first discuss the calculation of strain along the diffracting plane normal $N_{D}$ under Reuss assumption. The orientation of a crystallite of the $\varphi$ group with respect to the crystallographic axes, $x_{1}^{\prime \prime}, x_{1}^{\prime \prime}$, and $x_{3}^{\prime \prime}$ is shown in Fig. 2. The orientations of all other crystallites of the $\varphi$ group are obtained by varying angle $\varphi$ between $0^{\circ}$ and $180^{\circ}$. Another set of orthogonal axes $\left(x_{1}^{\prime}, i=1,2,3\right)$ is chosen such that $x_{3}^{\prime}$ is along the diffracting-plane normal $N_{D}$ and $x_{1}^{\prime}$ is along $O_{1} A$. The axis $x_{2}^{\prime}$ is not marked in Fig. (2). The stress components are referred to an orthogonal set of axes $\left(x_{i}\right)$ such that $x_{3}$ is along the direction of stress $\sigma$ and $x_{1}$ is in the plane $A B C$ and makes an angle $\varphi$ with $O_{1} A$. The angle between $x_{3}$ and $x_{3}^{\prime}$ is $(\pi / 2-\psi)$. The strain component along the plane normal $N_{D}$ is $\epsilon_{33}$ and can be calculated by the method detailed in an earlier article. ${ }^{17}$ Briefly, this is done as follows. The stress tensor $\sigma_{i j}$ is transformed to $\sigma_{i j}^{\prime \prime}$ using the relations

$$
\begin{gathered}
\sigma_{i j}^{\prime}=a_{i k} a_{j l} \sigma_{k l}, \\
\sigma_{i j}^{\prime \prime}=b_{i k} b_{j l} \sigma_{k l}^{\prime} .
\end{gathered}
$$

the term $a_{i j}$ denotes the matrix of the cosines of the angles which $x_{i}^{\prime}$ axes make with $x_{i}$ axes. Similarly, $b_{i j}$ denotes the matrix of the cosines of the angles which $x_{i}^{\prime \prime}$ axes make with $x_{i}^{\prime}$ axes. These matrices are given in an earlier article. ${ }^{17}$ The strain components produced by $\sigma_{g}^{\prime \prime}$ are given by

$$
\epsilon_{i j}^{\prime \prime}=S_{i j k l} \sigma_{k l}^{\prime \prime} \text {. }
$$

The strain component $\epsilon_{33}^{\prime}$ is given by

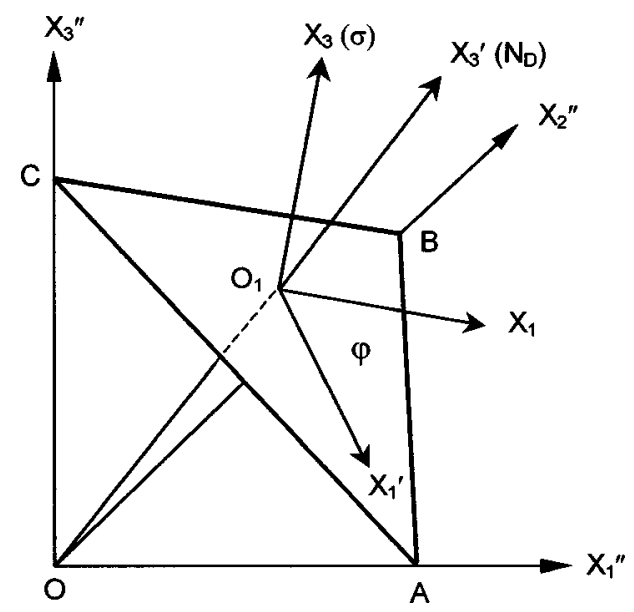

FIG. 2. $A B C$ indicates a crystallographic plane $(h k l) . N_{D}$ and $\sigma$ are normal to the plane $(h k l)$, and the applied stress, respectively. The angles between $O_{1} A$ and $O_{1} N_{D}$ and $\sigma$ are denoted by $\varphi$ and $(\pi / 2-\psi)$, respectively.

$$
\epsilon_{33}^{\prime}=\epsilon_{i j}^{\prime \prime} l_{i} l_{j},
$$

where $l_{i}$ denote the cosines of the angles $x_{3}^{\prime}$ makes with $x_{i}^{\prime \prime}$. Denoting $\epsilon_{33}^{\prime}$ by simply $\epsilon_{R}(\varphi)$ we get,

$$
\begin{aligned}
{\left[\epsilon_{R}(\varphi) / \sigma\right]=} & \left(S_{11} \sin ^{2} \psi+S_{12} \cos ^{2} \psi\right)+S\left(A_{1} \sin ^{2} \psi\right. \\
& -A_{1} \cos ^{2} \psi \cos ^{2} \varphi+A_{2} \cos ^{2} \psi \cos 2 \varphi \\
& +A_{3} \sin 2 \psi \cos \varphi+A_{4} \cos ^{2} \psi \sin 2 \varphi \\
& \left.+A_{5} \sin 2 \psi \sin \varphi\right),
\end{aligned}
$$

where $\psi$ denotes the angle between the diffracting plane normal and specimen surface normal. ${ }^{26}$ Other terms in Eq. (4) denote the following:

$S=S_{11}-S_{12}-S_{44} / 2$,

$A_{1}=-2\left(h^{2} k^{2}+k^{2} l^{2}+l^{2} h^{2}\right) /\left(h^{2}+k^{2}+l^{2}\right)^{2}$,

$A_{2}=-2 k^{2} l^{2} /\left(h^{2}+k^{2}+l^{2}\right)\left(k^{2}+l^{2}\right)$,

$A_{3}=\left[h^{3}\left(k^{2}+l^{2}\right)-h\left(k^{4}+l^{4}\right)\right] /\left(h^{2}+k^{2}+l^{2}\right)^{2}\left(k^{2}+l^{2}\right)^{1 / 2}$,

$A_{4}=h k l\left(k^{2}-l^{2}\right) /\left(h^{2}+k^{2}+l^{2}\right)^{3 / 2}\left(k^{2}+l^{2}\right)$,

$A_{5}=-k l\left(k^{2}-l^{2}\right) /\left(h^{2}+k^{2}+l^{2}\right)^{3 / 2}\left(k^{2}+l^{2}\right)^{1 / 2}$,

where $(h k l)$ are the Miller indices. This approach is general and can be used for a complex stress system. ${ }^{17} \mathrm{~A}$ simpler derivation for uniaxial stress can be found elsewhere. 9 The average of the strains in the $\varphi$ group of crystallites can be obtained from Eq. (4) by assuming a random orientation of crystallites in the sample and making use of the relations, $\left\langle\cos ^{2} \varphi\right\rangle=\left\langle\sin ^{2} \varphi\right\rangle=1 / 2$ and $\langle\cos n \varphi\rangle=\langle\sin n \varphi\rangle=0$, where $n$ is an integer and the angle brackets denote average over all orientations of $\varphi$. The average strain is given by

$$
\begin{aligned}
{\left[\left\langle\epsilon_{R}(\varphi)\right\rangle / \sigma\right]=} & \left(S_{11} \sin ^{2} \psi+S_{12} \cos ^{2} \psi\right) \\
& -\left(S A_{1} / 2\right)\left(1-3 \sin ^{2} \psi\right) .
\end{aligned}
$$

The strain under Voigt assumption is independent of $\varphi$ and is of the form ${ }^{4,9}$

$$
\left(\epsilon_{V} / \sigma\right)=X_{0 V}+X_{W} \sin ^{2} \psi
$$

The constants in Eq. (6) are given by ${ }^{4,9}$

$$
\begin{aligned}
& X_{0 V}=\frac{S_{11}\left(2 S_{11}+2 S_{12}-S_{44}\right)+S_{12}\left(3 S_{44}-4 S_{12}\right)}{2 S_{44}+6\left(S_{11}-S_{12}\right)}, \\
& X_{W}=\frac{5\left(S_{11}-S_{12}\right) S_{44}}{2 S_{44}+6\left(S_{11}-S_{12}\right)} .
\end{aligned}
$$

In an actual case, the measured strain lies between $\left\langle\epsilon_{R}(\varphi)\right\rangle$ and $\epsilon_{V}$. The theoretical estimate of the strain that would correspond to the measured strain from the line shift is given by

$$
\left\langle\epsilon(\varphi\rangle=\alpha\left\langle\epsilon_{R}(\varphi)\right\rangle+(1-\alpha) \epsilon_{V},\right.
$$

where $\alpha$ is a fraction lying between 0 and 1. It can be easily shown by combining Eqs. (5), (6), and (9a) that a plot of $\langle\epsilon(\varphi)\rangle$ versus $\sin ^{2} \psi$ is a straight line. The intercept and slope of the line are given, respectively, by

$$
X_{0}=\alpha\left(S_{12}-S A_{1} / 2\right)+(1-\alpha) X_{0 V}
$$


and

$$
X_{1}=\alpha\left(S_{11}-S_{12}+3 S A_{1} / 2\right)+(1-\alpha) X_{W} .
$$

The variance of strain is given by

$$
W_{\epsilon}=\left\langle\epsilon^{2}(\varphi)\right\rangle-\langle\epsilon(\varphi)\rangle^{2} .
$$

Since $\epsilon_{V}$ is independent of $\varphi$, Eq. (10) gives

$$
W_{\epsilon}=\left\langle\epsilon_{R}^{2}(\varphi)\right\rangle-\left\langle\epsilon_{R}(\varphi)\right\rangle^{2}
$$

On substituting for $\epsilon_{R}(\varphi)$ from Eq. (4) in Eq. (11) and carrying out the averages of the terms in $\varphi$ we get

$$
W_{\epsilon}=\alpha^{2} \sigma^{2} S^{2} F,
$$

where

$$
\begin{aligned}
F= & \frac{1}{2}\left(h^{2}+k^{2}+l^{2}\right)^{-4}\left\{\left[\left(h^{2} k^{2}+k^{2} l^{2}+l^{2} h^{2}\right)^{2}\right.\right. \\
& \left.-3 h^{2} k^{2} l^{2}\left(h^{2}+k^{2}+l^{2}\right)\right] \cos ^{4} \psi+\left[h^{6}\left(k^{2}+l^{2}\right)\right. \\
& +k^{6}\left(l^{2}+h^{2}\right)+l^{6}\left(h^{2}+k^{2}\right)-2\left(h^{4} k^{4}+k^{4} l^{4}\right. \\
& \left.\left.\left.+l^{4} h^{4}\right)\right] \sin ^{2} 2 \psi\right\} .
\end{aligned}
$$

\section{B. Variance from the measured profiles}

The measured line profile $h$ is given by the convolution of the standard profile $g$ with the profile $f$, which is produced by the defect structure (elastic strain in the present study). Langford $^{27}$ showed that these profiles could be represented by Voigt function. The Cauchy and Gaussian components of the profile are then given by

$$
h_{C}=g_{C}^{*} f_{C} \text { and } h_{G}=g_{G}^{*} f_{G} .
$$

The subscripts $C$ and $G$ denote the Cauchy and Gaussian components of the respective Voigt profiles. The asterisks denote the convolution of two functions. The following relations connect the integral widths of the profiles:

$$
\beta_{C}^{f}=\beta_{C}^{h}-\beta_{C}^{g} \text { and }\left(\beta_{G}^{f}\right)^{2}=\left(\beta_{C}^{h}\right)^{2}-\left(\beta_{G}^{g}\right)^{2} .
$$

In general, the Cauchy and Gaussian components of a profile are unique functions ${ }^{27}$ of the ratio $(2 w / \beta)$, where $2 w$ and $\beta$ are the full width at half maximum intensity (FWHM) and the integral width of the profile, respectively. De Keijser et $a .^{28}$ fitted the relations of the following form to the numerical data obtained from rigorous calculations: $:^{27}$

$$
\begin{aligned}
& \beta_{C} / \beta=\alpha_{0}+\alpha_{1} m+\alpha_{2} m^{2}, \\
& \beta_{G} / \beta=b_{0}+b_{1 / 2}(m-2 / \pi)^{1 / 2}+b_{1} m+b_{2} m^{2},
\end{aligned}
$$

where

$$
\begin{aligned}
& m=2 w / \beta, \quad \alpha_{0}=2.0207, \quad \alpha_{1}=-0.4803, \\
& a_{2}=-1.7756, \quad b_{0}=0.6420, \quad b_{1 / 2}=1.4187, \\
& b_{1}=-2.2043, \quad b_{2}=1.8706 .
\end{aligned}
$$

The maximum deviation between the values given by Eqs. (16) and (17), and the corresponding numerical values ${ }^{27}$ is about $1 \%$. Equations (16)-(18) can be used to obtain the Cauchy and Gaussian components of the $h$ and $g$ profiles. The Cauchy and Gaussian components of the $f$ profile can then be obtained by using Eq. (15). The apparent crystallite (domain) size is given by

$$
D=\lambda / \beta_{C}^{f} \cos \theta .
$$

The integral breadth $\beta$ is measured on the $2 \theta$ scale. $\lambda$ and $\theta$ are the wavelength and peak position of the diffraction line, respectively. The strain is given by

$$
e=\beta_{G}^{f} / 4 \tan \theta .
$$

The variance of strain is given by ${ }^{29,30}$

$$
W_{\epsilon}=1.5625 e^{2} \text {. }
$$

\section{Separation of $\alpha 1$ and $\alpha 2$}

The diffraction line profiles are often recorded with $K \alpha$ radiation. This gives rise to overlapping $\alpha 1$ and $\alpha 2$ lines. In the present work $\alpha 1$ line was separated by fitting the pseudoVoigt function $^{31}$ to the measured profile data. The measured profile is expressed as the superposition of the $K \alpha 1$ and $K \alpha 2$ profiles,

$$
I(2 \theta)=I_{1}(2 \theta)+I_{2}(2 \theta)+c_{0}+c_{1}(2 \theta)^{2} .
$$

$I_{1}(2 \theta)$ and $I_{2}(2 \theta)$ are the $K \alpha 1$ and $K \alpha 2$ profiles, respectively. The last two terms represent linearly varying background. $I_{1}(2 \theta)$ is represented by a pseudo-Voigt function of the form given later. A similar function holds good for $I_{2}(2 \theta)$ :

$$
I_{1}(2 \theta)=I_{1}^{0}\left[\eta L_{1}(2 \theta)+(1-\eta) G_{1}(2 \theta)\right],
$$

where $L_{1}(2 \theta)$ and $G_{1}(2 \theta)$ are the Cauchy and Gaussian functions given by

$$
L_{1}(2 \theta)=\left[1+\left(2 \theta-2 \theta_{1}\right)^{2} / w_{1}^{2}\right]^{-1},
$$

and

$$
G_{1}(2 \theta)=\exp \left[-(\ln 2)\left(2 \theta-2 \theta_{1}\right)^{2} / w_{1}^{2}\right] .
$$

In these equations, $2 \theta_{1}$ is the centroid position, which coincides with the profile maximum for symmetric profiles. $\eta$ is the mixing parameter, which determines the fractions of Cauchy and Gaussian components. $I_{1}^{0}$ is the peak intensity of the $\alpha 1$ diffraction line. The integral width is given by

$$
\beta_{1}=w_{1}\left[\eta \pi+\left(1-\eta(\pi / \ln 2)^{1 / 2}\right] .\right.
$$

The variances of the $\alpha 1$ diffraction lines were obtained by using the $2 w_{1}$ and $\beta_{1}$ values in place of $2 w$ and $\beta$ in Eqs. (15)-(20), respectively.

\section{EXPERIMENTAL DETAILS}

The polycrystalline samples of beta brass were prepared by carefully melting, under flux, the appropriate amounts of high purity copper and zinc, and casting. Attempts were made to achieve a composition with 52.8 wt \% copper and 47.2 wt \% zinc. This composition was important because the single-crystal elasticity data, required for the theoretical estimates of variances [Eq. (12)], are available only for this composition (Table I). The beta-brass casting was forged and hot rolled to give a 1-mm-thick strip. The dimensions of the tensile-test specimen prepared from this strip are shown in Fig. 1. Both the surfaces of the tensile-test specimen were cleaned and polished. The specimen was then annealed at $450 \mathrm{~K}$ for $72 \mathrm{~h}$ before recording the diffraction profiles. The 
TABLE I. Elasticity data of beta brass (body-centered-cubic, $\alpha=0.29587 \mathrm{~nm}$ ).

\begin{tabular}{|c|c|c|c|c|c|c|c|c|c|c|}
\hline \multicolumn{4}{|c|}{ Single crystal data ${ }^{\mathrm{a}}$} & \multicolumn{7}{|c|}{ Polycrystal data } \\
\hline & 11 & 12 & 44 & & V & $H$ & $S$ & $R$ & $A v .^{d}$ & Meas. ${ }^{\mathrm{e}}$ \\
\hline$C^{\mathrm{b}}$ & 1.2904 & 1.0956 & 0.8245 & $E^{\mathrm{c}}$ & 1.388 & 1.148 & 0.805 & 0.586 & 0.976 & 0.91 \\
\hline$S^{\mathrm{c}}$ & 3.5181 & -1.6154 & 1.2129 & $\nu$ & 0.301 & 0.335 & 0.384 & 0.416 & 0.359 & 0.28 \\
\hline \multicolumn{11}{|c|}{$\begin{array}{l}{ }^{a} \text { Reference } 32 \text {, sample composition-copper }(51.06 \text { wt } 5 \%) \text { and zinc }(48.94 \text { wt \%). } \\
{ }^{b} \text { Unit-Mb. } \\
{ }^{c} \text { Unit- }(\mathrm{Mb})^{-1} \text {. } \\
{ }^{\mathrm{d}} \text { Average of } \mathrm{H} \text { and } \mathrm{S} \text {. }\end{array}$} \\
\hline
\end{tabular}

copper-zinc ratio in the alloy was determined at a number of locations by energy dispersive $\mathrm{x}$-ray analysis. To assess the extent of texturing in the test specimen, three records of the (310) line were made on film using $\mathrm{Cu} K \alpha$ with the x-ray beam incident on the surfaces marked A, B, and C in Fig. 1.

The test specimen was loaded in tension in a specially designed jig that could be mounted on an automated Philips powder diffractometer. The macroscopic strain in the specimen produced by the applied load was measured using a strain gauge fixed on the specimen. Initially the specimen was loaded to indicate a strain level of $50 \times 10^{-6}$. This was taken as zero strain level. The diffractometer was set at $\psi=0$, and (310) diffraction line profile was recorded in the $2 \theta$ range $142^{\circ}-150^{\circ}$ with $\mathrm{Co} K \alpha$ radiation $\left(5^{\circ}\right.$ takeoff angle and $1^{\circ}$ divergence angle of the incident beam). The point-count method was employed using $40 \mathrm{~s}$ counting time at each step and the step intervals of $0.02^{\circ}(2 \theta)$. The measurement of line profile was repeated at five $\psi$ angles that corresponded to $\sin ^{2} \psi=0.1,0.2,0.3,0.4$, and 0.5 . The load was then increased until the strain level of $1.05 \times 10^{-3}$ was reached and the measurements of diffraction line profiles were repeated for all the $\psi$ angles mentioned earlier. Using $\mathrm{Cu} K \alpha$ radiation, the diffraction line profiles of (222) reflection were recorded in the range $127^{\circ}-131^{\circ}(2 \theta)$ at the strain levels 50 $\times 10^{-6}$ and $1.05 \times 10^{-3}$. At each strain level, the measurements were made at $\psi$ angles that corresponded to $\sin ^{2} \psi$ $=0,0.1,0.2,0.3$, and 0.4.

After each set of measurements at strain level of 1.05 $\times 10^{-3}$, the specimen was fully unloaded and the strain indicated by the strain gauge was noted. After recording of the x-ray diffraction line profiles, the test specimen was loaded in a universal-testing machine (Instron 1175). The longitudinal (along the load direction) and transverse strains, as registered by two suitably oriented strain gauges, were recorded as a function of applied load. These data were used to determine the Young's modulus and Poisson's ratio of polycrystalline beta-brass sample.

\section{RESULTS AND DISCUSSION}

\section{A. General}

While attempting to measure the broadening caused by the elastic anisotropy, it is important to eliminate other sources of broadening in the experiments. The major source, extraneous to the present context, is plastic deformation of the sample, ${ }^{1,2}$ which is known to produce changes in the sample that cause line broadening. For this reason, it is essential to avoid plastic deformation of the test specimen while loading. However, a high stress $\sigma$ is desirable to produce large broadening [Eq. (12)]. The maximum stress that can be applied without introducing plastic deformation is limited by the yield stress of the material. The beta-brass samples in the present experiments were found to be brittle and a reasonable estimate of the yield stress could not be made from the mechanical tests. We limited arbitrarily the maximum strain to $10^{-3}$. The stress corresponding to this strain, calculated using the measured Young's modulus (91.0 $\mathrm{GPa}$ ) is $0.091 \mathrm{GPa}$. The strain gauge showed nearly zero strain $\left( \pm 20 \times 10^{-6}\right)$ on releasing the load. This was taken to indicate the absence of any permanent (plastic) deformation in the sample during loading.

Other parameters which influence the broadening are $S$, $\alpha$, and $F$ [Eq. (12)]. We chose beta brass for the present study because it has a large value of $S$ (Table I). Since the factors affecting the value of $\alpha$ are not well understood, no step could be taken to maximize $\alpha$. In numerous studies made earlier (see Ref. 33, and references cited therein), $\alpha$ has been taken as an empirical parameter. In this study also, we treat $\alpha$ as an empirical parameter to be adjusted while comparing experimental results with the theoretical predictions. The term $F$ describes purely the dependence of the variance on $(h k l)$ and $\psi$ [Eq. (13)]. The $F$ versus $\sin ^{2} \psi$ plots for a few reflections are shown in Fig. 3. The $F$ values (and, therefore, the variances) for (420) reflection at all $\psi$ values between $0^{\circ}$ and $45^{\circ}$ are larger than the corresponding $F$ values for (310) reflection. Still, we chose (310) reflection in this work be-

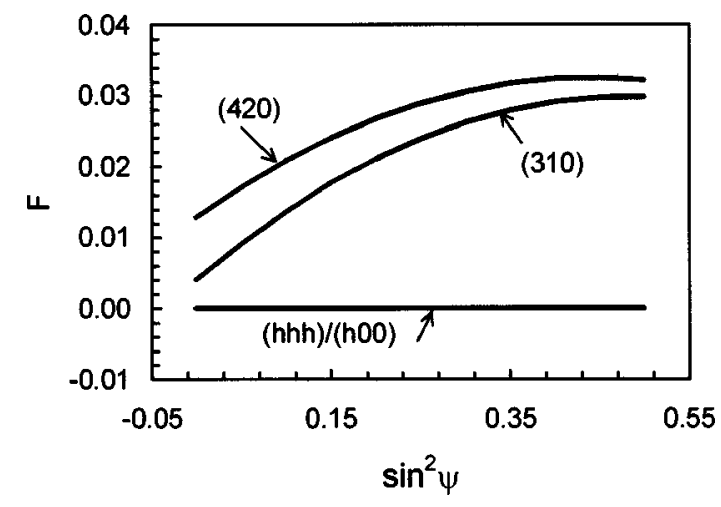

FIG. 3. The $F$ vs $\sin ^{2} \psi$ curves for (420), (310), and (hhh)/(h00)-type reflections. 

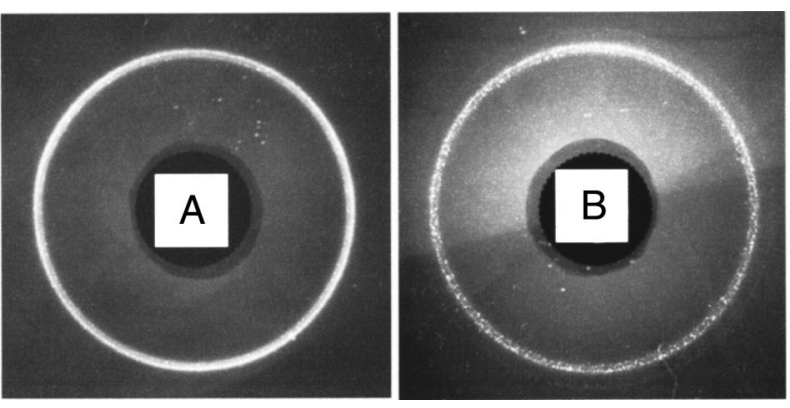

FIG. 4. Records of (310) lines (Co $K \alpha$ radiation) from surfaces A and B of the test specimen [Fig. (1)]. The record from surface $\mathrm{C}$ is similar to that from B

cause it occurs at large $2 \theta$ value $\left(\sim 145.9^{\circ}\right)$ with Co $K \alpha$ radiation. The wavelengths covered by the commercially available sealed x-ray tubes do not give (420) reflection at high $2 \theta$. It is interesting to note that $F$ vanishes for all values of $\psi$, for reflections of the type $(h h h)$ and $(h 00)$. To verify this trend, we chose (222) reflection.

The equations in Sec. II are derived assuming a random orientation of the crystallites in the sample. The preparation of the test specimen involved a number of thermomechanical steps that are likely to influence the orientation of the crystallites. Figure 4 shows the backreflection records of the (310) reflection taken from the surfaces A and B of the test specimen [Fig. (1)]. A reasonably uniform distribution of intensity along the diffraction ring in both the records indicates the absence of a pronounced texturing. Typical recorded diffraction profiles and the $\alpha 1-\alpha 2$ profiles separated using Eq. (22) are shown in Fig. 5. The typical widths (FWHM) of $\alpha 1$ components of these profiles are $\approx 1^{\circ}$, as compared to $\approx 0.3^{\circ}$ for the profile recorded with fully annealed $\approx 80 \mu$ beta-brass powder. A sharper diffraction line from the bulk specimen is preferable. However, attempts to increase the grain size in the bulk specimen through increased annealing time or temperature resulted in grain structure that produced spottiness and uneven intensity distribution in the diffraction ring. This resulted in intensity fluctuations in the diffraction profiles. The selection of the present sample was based on a compromise between the linewidth and smoothness of the intensity in the diffraction ring.

\section{B. Analysis of the line shift (average strain)}

The lattice strains calculated from the line shift in the loaded test specimen $(\sigma=0.091)$ as a function of $\sin ^{2} \psi$ are shown in Fig. 6. As expected from Eqs. (9a)-(9c), a straight line fits the data very well. The intercept and slope of the line are -0.0058 and $0.0183 \mathrm{GPa}^{-1}$, respectively. These values can be reproduced using single crystal elastic moduli (Table I) and $\alpha=0.3$ in Eqs. (9b) and (9c). These results are in good agreement with earlier results of Macherauch, ${ }^{33}$ who obtained $0.00608,0.017 \mathrm{GPa}^{-1}$, and 0.33 for the intercept, slope, and $\alpha$, respectively.

\section{Analysis of the broadening (variance of strain)}

The parameter $2 w_{1} / \beta_{1}$ as a function of $\sin ^{2} \psi$ for the (310) $\alpha 1$ profile is shown in Fig. 7. At all $\sin ^{2} \psi$ values, the
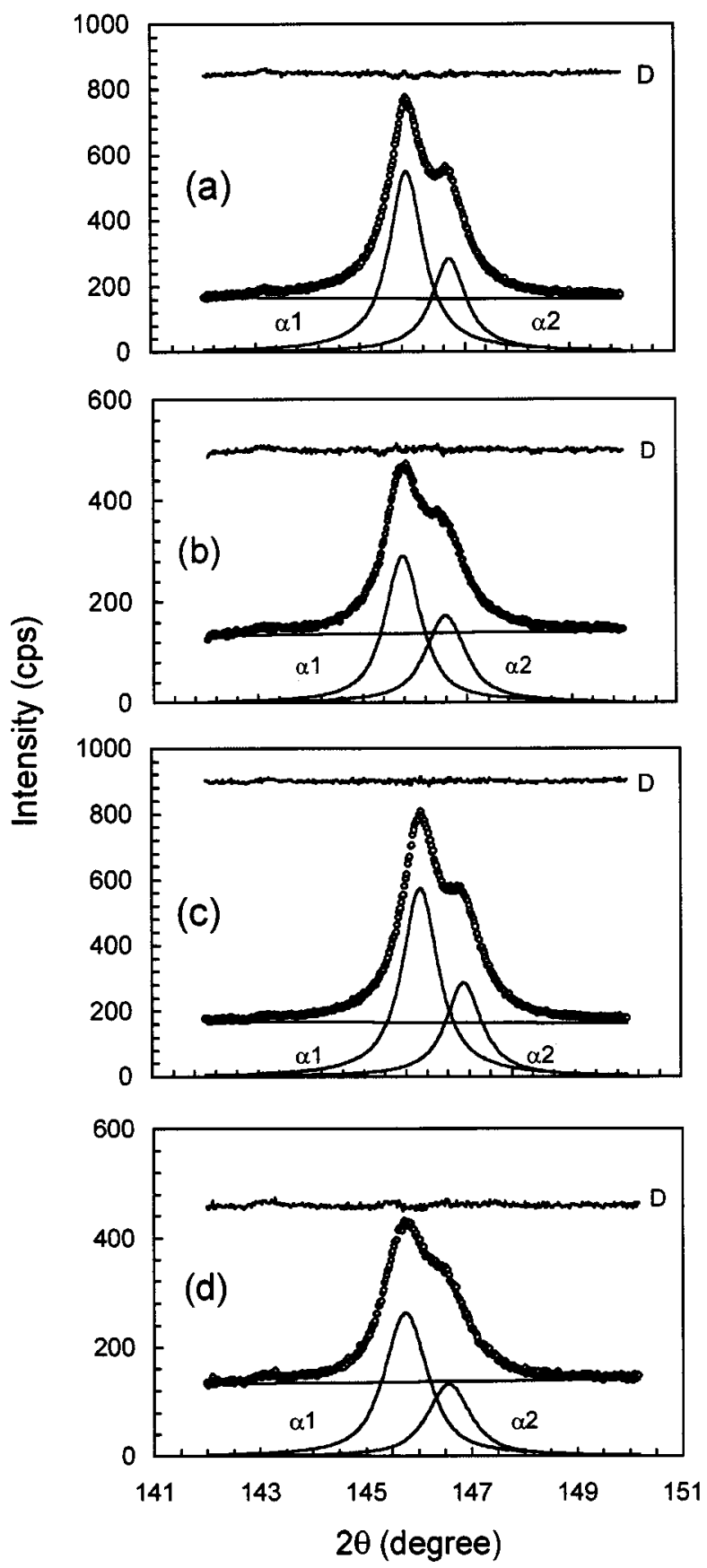

FIG. 5. The as-recorded (310)-line profiles of beta brass taken with Co $K \alpha$ radiation and separated $\alpha 1-\alpha 2$ doublets under different experimental conditions. No-load: (a) and (b) at $\psi=0^{\circ}$ and $\psi=45^{\circ}$, respectively. Under load: (c) and (d) at $\psi=0^{\circ}$ and $\psi=45^{\circ}$, respectively. The lines marked D (arbitrarily shifted along $y$ axis for clarity) show the differences between the observed and fitted profiles. The typical values of the standard errors in $2 w_{1}$ and $2 \theta_{1}$ (both in degrees) are 0.002 and 0.001 , respectively.

parameter $2 w_{1} / \beta_{1}$ is found to increase appreciably on application of load. The variances at different $\sin ^{2} \psi$ values were derived from the $2 w_{1} / \beta_{1}$ values using Eqs. (15), (17), and (20). These values together with the values calculated from Eq. (12) are shown in Fig. 8. The best agreement between the variances obtained from line profile analysis and those calculated from Eq. (12) is obtained with $\alpha=0.5 \pm 0.06$.

The apparent crystallite (domain) size can be calculated using Eq. (19). The Cauchy component $\beta_{1 C}^{f}$ of the width for 


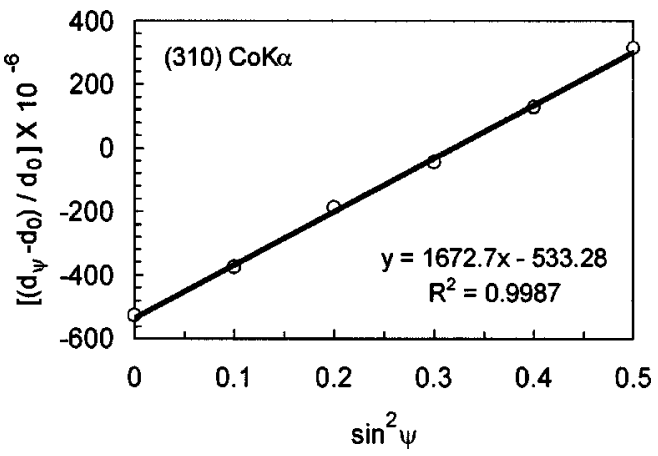

FIG. 6. The lattice strain as a function of $\sin ^{2} \psi$ for an applied stress 0.091 $\mathrm{GPa}$. The error bars are the size of the symbols.

(310) $\alpha 1$ profile did not show any systematic dependence on $\sin ^{2} \psi$. The average values of $\beta_{1 C}^{f}$ (on $2 \theta$ scale) were 1.24 $\pm 0.04^{\circ}$ and $1.22 \pm 0.02^{\circ}$ for the no-load and loaded conditions, respectively. After correcting for instrumental broadening, these data gave $43.3 \pm 0.6 \mathrm{~nm}$ for the crystallite size. Within the experiment error, the crystallite size remains unchanged on application of load.

The $2 w_{1} / \beta_{1}$ values for the (222) $\alpha 1$ line under different experimental conditions are shown in Fig. 9. Within the experimental errors, $2 w / \beta$ is not effected by the application of load. This suggests that the (222) line does not broaden on application of load. This trend is predicted by Eq. (12)

The broadening of the $\mathrm{x}$-ray powder diffraction lines under nonhydrostatic compression in an opposed anvil device has been investigated recently. ${ }^{25}$ This work discusses theories based on uniaxial stress and local stress models. The theory in the framework of uniaxial stress model is an extension of earlier work. ${ }^{17,20}$ The results of this model correspond to the broadening discussed in the present work. The main conclusions of this analysis ${ }^{25}$ are: (1) the lines do not broaden if the sample material is elastically isotropic, (2) the broadening vanishes for the reflections of the type $(h 00)$ and (hhh), and (3) broadening vanishes for all (hkl) at $\psi=\pi / 2$ (note the redefinition ${ }^{26}$ of $\psi$ in the present work). It can be easily verified that these trends are also predicted by Eq. (12). The local stress model ${ }^{25}$ suggests that the line width of (hkl) depends linearly on $1 / E(h k l)$, where $E(h k l)$ is the Young's modulus along the direction $(h k l)$. The measured

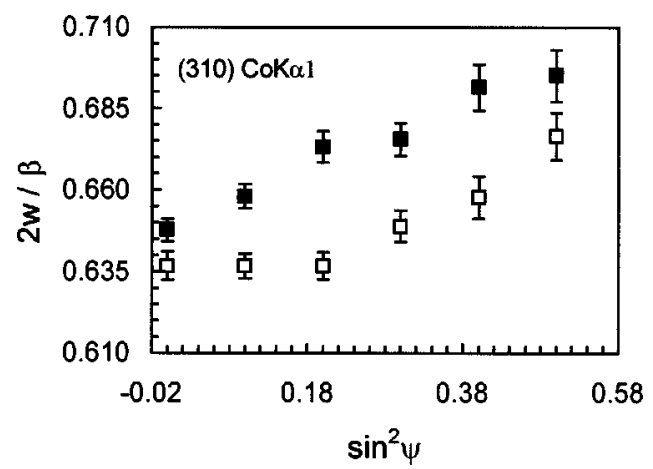

FIG. 7. The parameter $2 w_{1} / \beta_{1}\left(2 \theta^{\circ}\right.$ scale) as function of $\sin ^{2} \psi$ for (310) reflection. Without load - open squares. With load $(\sigma=0.091 \mathrm{GPa})-$ filled squares.

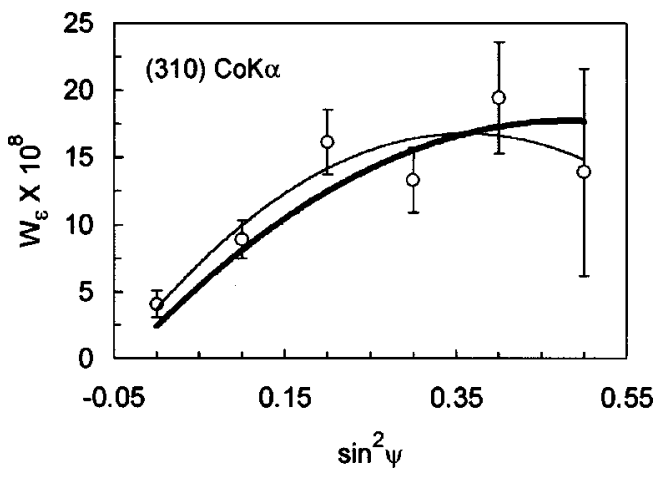

FIG. 8. A comparison of the measured variance from the line profile analysis (open circles and the best-fit parabola shown by a thin line) with the variance (thick line) calculated from Eq. (7) with $\alpha^{2}=0.25 \pm 0.05$.

linewidths for gold at $77 \mathrm{GPa}$ follow this trend closely. Small deviations of the (220) and (311) data points from this trend are attributed to the contribution from uniaxial stress model. This provides only a qualitative evidence for the line broadening in the framework of uniaxial stress model.

\section{SUMMARY}

The expressions for the average lattice strain and the variance of lattice strain for a polycrystalline sample (cubic system) under an uniaxial elastic deformation have been derived. We chose beta brass to test the theoretical predictions, as it exhibits large elastic anisotropy. The diffraction profiles of (310) and (222) of beta brass were recorded using Co $K \alpha$ and $\mathrm{Cu} K \alpha$ radiation, respectively. The lattice strain derived from the line shift depends linearly on $\sin ^{2} \psi$, as predicted by the theory. The slope and intercept of the strain versus $\sin ^{2} \psi$ line agree very well with the results of an earlier study. The $\sin ^{2} \psi$ dependence of the variance for (310) reflection predicted by theory is supported by the experimental results. The theoretical prediction that the reflections of the type $(h h h)$ do not broaden under elastic deformation is supported by the line profile data on (222) reflection. The line broadening arising from elastic anisotropy alone is indeed small. However, since the extent of broadening depends on the magnitude of uniaxial stress, this effect is expected to become appreciable in the high-pressure $\mathrm{x}$-ray diffraction data of materials that can support large uniaxial stress component.

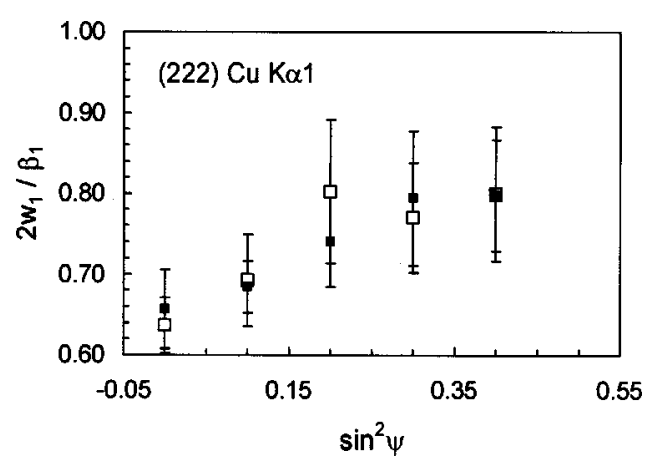

FIG. 9. The parameter $2 w_{1} / \beta_{1}\left(2 \theta^{\circ}\right.$ scale $)$ as function of $\sin ^{2} \psi$ for (222). Without load - open square. With local $(\sigma=0.091 \mathrm{GPa})-$ filled squares. 


\section{ACKNOWLEDGMENTS}

The authors thank D. Dwarakanatha Rao for the help in preparation of beta-brass samples. One of the authors (A.K.S.) acknowledges the support under CSIR emeritus scientist scheme, and thanks Ken-ichi Kondo for the invitation to the Tokyo Institute of Technology, Yokohama, Japan, where this article was completed.

${ }^{1}$ C. S. Barrett and T. B. Massalski, Structure of Metals, 3rd ed. (McGrawHill, New York, 1966)

${ }^{2}$ H. P. Klug and I. Alexander, X-Ray Diffraction Procedures for Polycrystalline and Amorphous Materials, 2nd ed. (Wiley, New York, 1974).

${ }^{3}$ B. D. Cullity, Elements of X-Ray Diffraction, 2nd ed. (Addison-Wesley, Reading, MA, 1978)

${ }^{4}$ I. C. Noyan and J. B. Cohen, Resident Stress Measurement by Diffraction and Interpretation (Springer, New York, 1987).

${ }^{5}$ A. J. C. Wilson, Proc. Phys. Soc. London 80, 286 (1962).

${ }^{6}$ A. J. C. Wilson, Proc. Phys. Soc. London 81, 41 (1963).

${ }^{7}$ F. Weaver and B. Pfarr, Mitt. K. Wilh.-Inst. Eisenforsch. 15, 137 (1933).

${ }^{8}$ S. L. Smith and W. A. Wood, Proc. R. Soc. London, Ser. A 176, 398 (1940).

${ }^{9}$ G. B. Greenough, Prog. Met. Phys. 3, 176 (1951).

${ }^{10}$ C. M. Sayers, Philos. Mag. 49, 243 (1984).

${ }^{11}$ W. Voigt, Lehrbuch der Kristallphysik (Teubner, Leipzig, 1928).

${ }^{12}$ A. Reuss, Z. Angew. Math. Mech. 9, 49 (1929).

${ }^{13}$ E. Kröner, Z. Phys. 151, 504 (1958).

${ }^{14}$ A. K. Singh, and G. C. Kennedy, J. Appl. Phys. 45, 4686 (1974).
${ }^{15}$ A. L. Ruoff, J. Appl. Phys. 46, 1389 (1975).

${ }^{16}$ R. Jeanloz, B. K. Godwal, and C. Meade, Nature (London) 349, 687 (1991).

${ }^{17}$ A. K. Singh, J. Appl. Phys. 73, 4278 (1993); 74, 5920 (1993).

${ }^{18}$ Y. Meng, D. J. Weidner, and Y. Fei, Geophys. Res. Lett. 20, 1147 (1993).

${ }^{19}$ A. K. Singh and C. Balasingh, J. Appl. Phys. 75, 4327 (1994).

${ }^{20}$ T. Uchida, N. Funamori, and T. Yagi, J. Appl. Phys. 80, 739 (1996).

${ }^{21}$ R. J. Hemley et al., Science 276, 1242 (1997).

${ }^{22}$ A. K. Singh, C. Balasingh, H. K. Mao, R. J. Hemley, and J. Shu, J. Appl. Phys. 83, 7567 (1998).

${ }^{23}$ A. K. Singh, H. K. Mao, J. Shu, and R. J. Hemley, Phys. Rev. Lett. 80, 2157 (1998).

${ }^{24}$ T. S. Buffy et al., Phys. Rev. B 60, 15063 (1999).

${ }^{25}$ N. Funamori, M. Funamori, and R. Jeanloz, J. Appl. Phys. 82, 142 (1997).

${ }^{26}$ Following the nomenclature adopted in the literature on stress measurement, we use $\psi$ to denote the angle between the diffracting plane normal and specimen surface normal. In the lattice strain equations (Refs. 12-23), $\psi$ was used to denote the angle between the diffracting plane normal and the direction of applied load. The two choices of $\psi$ represent complementary angles.

${ }^{27}$ J. I. Langford, J. Appl. Crystallogr. 11, 10 (1978).

${ }^{28}$ Th. H. de Keijser, J. I. Langford, E. J. Mittemeijer, and A. B. P. Vogels, J. Appl. Crystallogr. 15, 308 (1982).

${ }^{29}$ A. R. Stokes and A. J. C. Wilson, Proc. Phys. Soc. London 56, 174 (1944).

${ }^{30}$ D. R. Buchanan and R. L. McCullough, and R. L. Miller, Acta Crystallogr. 20, 922 (1966).

${ }^{31}$ F. Sánchez-Bajo and F. L. Cumbrera, J. Appl. Crystallogr. 30, 427 (1997).

${ }^{32}$ D. Lazarus, Phys. Rev. 76, 545 (1949).

${ }^{33}$ E. Macherauch, Exp. Mech. 23, 140 (1966). 